\title{
Shifts between cooperation and antagonism driven by individual variation: a systematic synthesis review
}

Moran, Nicholas P.; Caspers, Barbara A.; Chakarov, Nayden; Ernst, Ulrich R.; Fricke, Claudia; Kurtz, Joachim; Lilie, Navina D.; Lo, Lai Ka; Müller, Caroline; Reshma, R.

Total number of authors:

15

Published in:

Oikos

Link to article, DOI:

10.1111/oik.08201

Publication date:

2022

Document Version

Publisher's PDF, also known as Version of record

Link back to DTU Orbit

Citation (APA):

Moran, N. P., Caspers, B. A., Chakarov, N., Ernst, U. R., Fricke, C., Kurtz, J., Lilie, N. D., Lo, L. K., Müller, C., Reshma, R., Takola, E., Trimmer, P. C., van Benthem, K. J., Winternitz, J., \& Wittmann, M. J. (2022). Shifts between cooperation and antagonism driven by individual variation: a systematic synthesis review. Oikos, 2022(1), [e08201]. https://doi.org/10.1111/oik.08201

\section{General rights}

Copyright and moral rights for the publications made accessible in the public portal are retained by the authors and/or other copyright owners and it is a condition of accessing publications that users recognise and abide by the legal requirements associated with these rights.

- Users may download and print one copy of any publication from the public portal for the purpose of private study or research.

- You may not further distribute the material or use it for any profit-making activity or commercial gain

- You may freely distribute the URL identifying the publication in the public portal 


\title{
$01 k \bigcirc S$
}

\section{Forum}

\section{Shifts between cooperation and antagonism driven by individual variation: a systematic synthesis review}

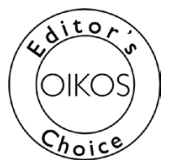

\author{
Nicholas P. Moran, Barbara A. Caspers, Nayden Chakarov, Ulrich R. Ernst, Claudia Fricke, Joachim Kurtz, \\ Navina D. Lilie, Lai Ka Lo, Caroline Müller, Reshma R, Elina Takola, Pete C. Trimmer, Koen J. van Benthem, \\ Jamie Winternitz and Meike J. Wittmann
}

N. P. Moran (https://orcid.org/0000-0002-7331-0400) $\square$ (nicholaspatrickmoran@gmail.com), Centre for Ocean Life DTU-Aqua, Technical Univ. of Denmark, Lyngby, Denmark. - NPM and and N. D. Lilie (https://orcid.org/0000-0002-3421-714X), Dept of Evolutionary Biology, Bielefeld Univ., Bielefeld, Germany. B. A. Caspers (https://orcid.org/0000-0002-4380-0476), Dept of Behavioural Ecology, Bielefeld Univ., Bielefeld, Germany. - N. Chakarov, NDL andJ. Winternitz (https://orcid.org/0000-0002-1113-9126), Dept of Animal Behaviour, Bielefeld Univ., Bielefeld, Germany. - U. R. Ernst (https://orcid.org/0000-0002-63305341), C. Fricke (https://orcid.org/0000-0002-0691-6779), J. Kurtz (https://orcid.org/0000-0002-7258-459X), L. K. Lo and R. R, Inst. for Evolution and Biodiversity, Univ. of Münster, Münster, Germany. URE also at: Apicultural State Inst., Univ. of Hohenheim, Stuttgart, Germany. - C. Müller (https:/lorcid. org/0000-0002-8447-534x), Dept of Chemical Ecology, Bielefeld Univ., Bielefeld, Germany. - E. Takola (https://orcid.org/0000-0003-1268-5513), Inst. of Ecology and Evolution, Friedrich Schiller Univ. Jena, Jena, Germany. - P. C. Trimmer, Dept of Psychology, Univ. of Warwick, Coventry, UK. - K. J. van Benthem (https:// orcid.org/0000-0002-3841-2110) and M. J. Wittmann (https:/lorcid.org/0000-0002-7209-9172), Dept of Theoretical Biology, Bielefeld Univ., Bielefeld, Germany.

\section{Oikos}

2022: e08201

doi: $10.1111 /$ oik.08201

Subject Editor: Matthew Symonds Editor-in-Chief: Dries Bonte Accepted 13 August 2021

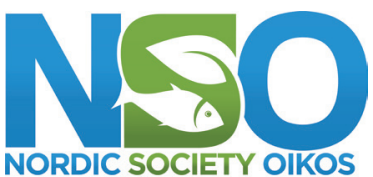

www.oikosjournal.org
The outcomes of interspecific and intraspecific ecological interactions can be considered to fall along continua from cooperative (mutually beneficial) to antagonistic (detrimental to one or both parties). Furthermore, the position of an interaction outcome along the continuum, for example whether a symbiont provides net costs or benefits to its host, or whether two conspecifics cooperatively forage or compete for food, is often not fixed but can change over time or across contexts. In this systematic review, we investigate the role of intraspecific trait variation ('ITV') in one or both interacting parties in determining the cooperative-antagonistic outcome of inter- and intraspecific ecological interactions. Based on a literature collection of 96 empirical and theoretical publications meeting our inclusion criteria, we give an overview of the types of interaction continua involved; traits related to outcome variance; and mechanisms as well as constraints on shifts in interactions outcomes. We propose that ITV can lead to shifts in interaction outcomes via two interrelated mechanisms. First, trait frequency effects occur when there are changes in a population's composition of traits linked to cooperation or antagonism (e.g. aggressive personality types, cheater phenotypes etc.), leading to net shifts in interaction outcomes. Second, systemic variance effects occur where the level of ITV in a trait in a population (as opposed to the

This systematic literature review synthesises knowledge on the processes through which an ecological interaction between individuals can shift between being mutually beneficial and detrimental to one or both parties. The article uniquely focuses on how these shifts can $\frac{\infty}{4}$ be caused by trait variation among individuals arising for example from heritable genetic variation or phenotypic plasticity. Such shifts occur in interactions between species (e.g. के plant-animal, host-symbiont) and within species (e.g. social groups, male-female, parentoffspring). Bringing together information from 96 empirical and theoretical publications across these various contexts, this review provides a conceptual framework for qualitative shifts in interaction outcomes mediated by intraspecific trait variation.

(C) 2021 The Authors. Oikos published by John Wiley \& Sons Ltd on behalf of Nordic Society Oikos. This is an open access article under the terms of the Creative Commons Attribution License, which permits use, distribution and reproduction in any medium, provided the original work is properly cited. 
mean value) is the factor that influences the cooperative-antagonistic outcome. Heritable trait differences and phenotypic plasticity are sources of phenotypic variation among individuals, and both the degree of heritability and plasticity of the trait involved may determine whether shifts between cooperation and antagonism are likely to be short-term (i.e. context-dependent) or lead to more persistent shifts (e.g. mutualism breakdown). To guide future research, we describe knowledge gaps and divergences between empirical and theoretical literature, highlighting the value of applying evidence synthesis methods in ecology and evolution.

Keywords: individualised niche, kinship, mutualism, personality, phenotypic plasticity, state-dependence

\section{Introduction}

Intraspecific trait variation ('ITV') is ubiquitous in nature. Variation among individuals of the same species arises through a combination of underlying genetic/epigenetic variation (e.g. variation in genotypes that may arise via mutation, selection and genetic drift), and phenotypic plasticity (i.e. where environmental factors influence trait expression, where both the factor driving the response and the response itself may vary at an individual level (Dewitt and Scheiner 2004). Thus, individual phenotypic traits fall along spectra of heritability and plasticity (Lynch and Walsh 1998, Bolnick et al. 2003). Phenotypic variation within species can occur at magnitudes comparable to variation between species and can have comparable effects on ecological processes, community composition and species richness (Des Roches et al. 2018). Trait differences can also be linked to intraspecific task specialisation and niche differentiation among individuals (Dall et al. 2012), i.e. the 'individualised niche' (Müller et al. 2020).

The level of ITV in a population can influence the outcome of ecological interactions via multiple mechanisms (Bolnick et al. 2011). For example, through nonlinear averaging, the realised strength of an interaction in the presence of variation may differ from the strength predicted based on the mean trait (Jensen's inequality, Jensen 1906). Similarly, different responses of individuals to environmental fluctuations buffer population dynamics against strong environmental stochasticity (the portfolio effect, Tilman et al. 1998). ITV can have direct ecological consequences that are independent of trait heritability, and also have more long-term implications for eco-evolutionary dynamics (Bolnick et al. 2011). Theoretical studies of ITV effects of ecological interactions have previously explored how ITV can alter the strength and stability of an interaction (Moran et al. 2017). For example, ITV can determine the superior competitor in an interspecific competitive interaction and may promote or hinder species coexistence (Hart et al. 2016, Uriarte and Menge 2018, Milles et al. 2020). However, in these models it is often assumed that, no matter what the level of variation is, the interaction stays competitive.

Here we explore whether and under what circumstances ITV can have even more drastic consequences and lead to qualitative changes in the outcome of an interaction. Ecological interactions fall along a two-dimensional continuum defined both by the fitness effect of party A on party B and by the effect of party B on party A. We specifically focus on cases where at least one of these mutual effects changes sign and changes the quality of the interactions from cooperative/mutualistic (i.e. providing beneficial outcomes to both parties) to antagonistic (i.e. detrimental to one or both parties, Bronstein 1994) or vice versa. The processes described in this review may also be relevant to cases where outcomes may vary without necessarily changing in sign (i.e. a cooperative interaction becoming more or less cooperative). Nonetheless, we have targeted our systematic review to specifically focus on interactions where net cooperative or antagonistic outcomes are able to occur, and we expect these shifts to have profound effects on the ecology of the system. Also, note that we adopt the term mutualism to refer to beneficial interspecific interactions, and cooperative to inclusively refer to beneficial interactions at inter- and intra-specific levels (as per West et al. 2007).

The position of any interaction along the continuum (or the cooperative-antagonistic 'quality' of the interaction) is not fixed; for example, biotic and abiotic environmental factors can change the net outcome of an interaction between positive and negative (e.g. 'context-dependent outcomes', as per Chamberlain et al. 2014; 'interaction norms', Thompson 1988). This outcome variance is relevant to interspecific interactions, which (non-exhaustively) includes beneficial versus exploitative animal-plant interactions (Morris et al. 2007, Anjos et al. 2020), mutualistic versus competitive, or predatory animal-animal interactions (Holland et al. 2005) or mutualistic versus parasitic host-symbiont interactions (Karst et al. 2008, Canestrari et al. 2014). Outcome variation has also been found in intraspecific interactions, where relevant types of interaction may include variation between cooperation and competition within socially interacting dyads or groups (Sachs et al. 2004), and between reproductive cooperation and conflict in female-male pairs or parent-offspring relationships (Arnqvist and Rowe 2005, Székely et al. 2007).

Variation in interaction outcomes may occur between individuals of the same species or over time (e.g. within-individual variation), which provides the material through which the net interaction quality for a species or population may change over time (e.g. 'evolution of interactions', Thompson 1988), potentially leading to longer-term evolutionary transitions (e.g. 'mutualism breakdown', Sachs and Simms 2006). Furthermore, shifts between cooperative-antagonistic outcomes appear to be relatively common over ecological time frames (Bronstein 1994, Chamberlain et al. 2014). These processes are essential to our understanding of ecological networks, as the balance of cooperation-antagonism within interaction networks may be closely associated with 
(A) Trait frequency effects, i.e. within-population changes in the composition of traits linked to cooperation-antagonism, e.g. due to selective or genetic drift effects, or phenotypic plasticity.

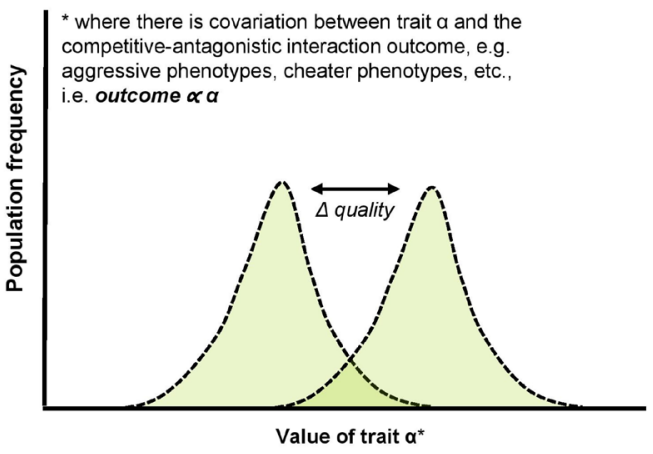

(B) Systemic variance effects, i.e. the level of trait variation within a population is linked to cooperation/antagonism, where greater ITV within a population can be associated with either more cooperative or more antagonistic outcomes.

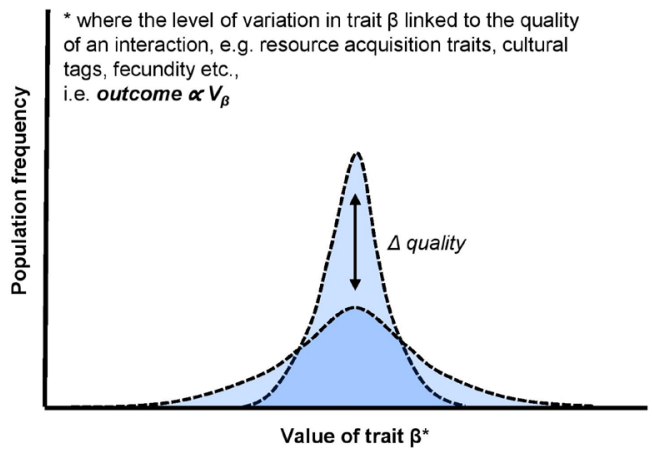

(C) Links between trait frequency and variance effects. Changes in the composition of cooperation-antagonism linked traits can directly lead to outcome variation (i.e. trait frequency effects, marked in green). Changes in trait variance can lead to systemic variance effects (marked in blue) by (i) directly producing outcome variation, e.g. by influencing the identity of interaction partners and altering their fitness payoffs; or, (ii) inducing changes in the composition of cooperation-antagonism linked traits, e.g. by inducing behavioural plasticity in those traits.

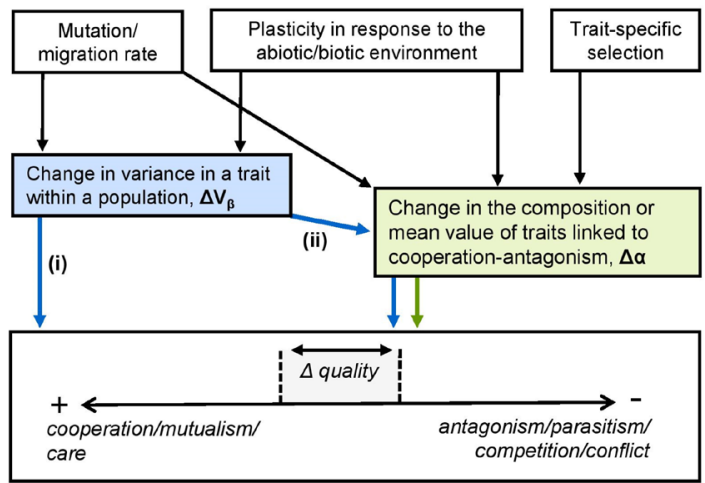

Figure 1. Conceptual diagrams showing changes in the cooperativeantagonistic quality of an interaction due to (A) trait frequency effects and (B) systemic variance effects. Diagram (C) shows some possible pathways where changes in trait variance and composition can lead to outcome variation, as encountered in the literature. Trait values $(\alpha, \beta)$ refer to an arbitrary measure of a hypothetical trait that varies within a population while $\Delta$ quality represents a change in the qualitative outcome of an interaction along a cooperative-antagonistic continuum. population and community stability (Mougi and Kondoh 2012, Montesinos-Navarro et al. 2017).

We propose that ITV in one or both interaction partners can affect shifts along cooperation-antagonism continua via two types of process (Fig. 1). The most direct way is if there is intraspecific variation in a trait that directly influences how cooperatively or antagonistically an individual acts in an interaction (i.e. trait-outcome covariation, such as in cooperative versus cheater phenotypes). Many distinct processes can influence the composition and frequency of these traits within a population, which can shift the net quality of an interaction between mutualism and antagonism (referred to here as trait frequency effects, Fig. 1A). For example, more cooperative or antagonistic phenotypes may be subject to trait-specific selection (Aukema and Raffa 2004), or individuals may show state- or condition- dependent expression of cooperativeantagonistic phenotypes in response to environmental factors (e.g. resource availability, Marshall et al. 2016, or physical conditions, Walker et al. 2007, Marshall et al. 2016).

In addition to these more straightforward processes, changes in the level of ITV within a population (as opposed to the mean value) can be a factor that has direct and indirect effects on the cooperative-antagonistic outcome of an interaction (referred to here as systemic variance effects, Fig. 1B). In this case, different levels of trait variation may lead to different outcomes, even where the trait may be seemingly unrelated to cooperation or antagonism. Trait variation can have direct effects on the net costs or benefits individuals derive from interactions (Fig. 1C, pathway i). For example, greater variation between individuals in traits related to resource acquisition or parental care can be more likely to lead to mutually beneficial outcomes in cooperative foraging or breeding interactions (Uitdehaag 2011, Barta 2016). The level of trait variance may also have more indirect effects on interaction outcomes by inducing a trait frequency effect (Fig. 1C, pathway ii). For example, the level of variation in fecundity among individuals in a conspecific group can affect patterns of relatedness in neighbourhoods, which in turn favours cooperative behaviours (Rodrigues and Gardner 2013).

Information on how ITV can lead to such qualitative shifts in an interaction is currently scattered in the literature and the phenomena outlined here can go by many names. Our overall goal was therefore to provide a conceptual synthesis of literature relating to the a priori defined research question: 'How can intraspecific trait variation shift an interaction along the gradient from antagonistic to mutualistic interactions, both for intraspecific and for interspecific interactions? For example, can intraspecific variation in hosts and/or parasites (individualised niches) change the relationship so that the net effect of the 'parasite' on the host becomes positive?' We aimed to synthesise both empirical and theoretical literature, and compare the coverage of both to identify understudied topics and provide direction for future studies. This review is based on a literature collection obtained primarily using a systematic literature search to establish an unbiased cross-sectional coverage of relevant studies, supplemented with additional nonsystematic articles to ensure comprehensive coverage. 


\section{Literature collection}

\section{Search strategy and protocol}

Our systematic search used a query composed of terms relating to (a) cooperative/mutualistic interactions, (b) antagonistic interactions and (c) intraspecific ecological variation (general query: (“*mutualis*” OR "cooperati*” OR "interdependenc" OR "symbio*") AND ("antagonis" OR "competi*” OR ("host*” AND "parasit*") OR ("predator*" AND "prey") OR "conflict") AND ((“intraspecific" OR "within-species" OR “individual*” OR "agent" mal*") NEAR/5 ("varia*” OR "divers*” OR “difference*”))). Searches were conducted in Web of Science (18 July 2019; refined to categories Ecology, Evolutionary biology, Zoology and Behavioural sciences) and Scopus (18 July 2019; refined to the subject area Agricultural and Biological sciences; see full search strategy in the Supporting information). Duplicates were removed via $\mathrm{R}$ package revtools (ver. 0.4.1, Westgate 2019) and manually, giving 411 unique records.

\section{Study selection}

Titles and abstracts of records were screened by two reviewers (from a pool of eight) to avoid individual selection biases, using randomised author-, journal- and metadatablind screening. Records were included for full-text screening when considered relevant, or potentially relevant, to our predefined research question (as stated above). Conflicting decisions $(140 / 411,34 \%)$ were resolved by a third reviewer. Following a preliminary assessment of each paper's topic, full texts were allocated to a reviewer (from a pool of 15) based on their expertise. A total of 192 full-text records were screened based on the criteria: 1) is the paper accessible in English or German?; 2) is there an interspecific or intraspecific interaction that can vary from cooperative/ mutualistic to antagonistic?; 3) is there trait variation?; 4) is the trait variation intraspecific?; and 5) does the ITV influence the mutualistic/antagonistic direction of the interaction? Variation in the strength of a cooperative interaction without evidence of corresponding variation in antagonism, or vice versa, was not considered as a shift in cooperativeantagonistic quality, i.e. there needed to be outcome variation in an interaction that could show both mutualistic and antagonistic qualities. Papers not meeting all criteria were excluded. Empirical studies with humans as the focal species were also excluded as beyond the scope of the review, although theoretical/modelling studies based on humans, but with potential general applicability could be included. In addition, one study was also included where humans were involved in the interaction but were not the focal species (Cantor et al. 2018). Full-text reviewer decisions were double-checked for consistency and conflicting decisions were resolved collaboratively (by NPM, MJW, include/exclude conflicts for $16 / 192$ papers, $8.3 \%$ ).

To complement this collection and ensure comprehensive coverage of the literature, non-systematically sourced papers meeting our criteria were also included from: 1) papers identified from the references of papers from the above systematic searches; and 2) relevant papers already known to authors. For included studies, we extracted at least: the study type (empirical, observational, review, theory/modelling); the scale of ecological interaction considered (interspecific, intraspecific, both); and, the type of ecological interactions considered (host-symbiont, cooperative-competitive, female-male, etc.). Review records following PRISMA guidelines are available in the Supporting information and on the Open Science Framework (<https://osf.io/9kfpc/>; doi: 10.17605/OSF. IO/9KFPC; Moher et al. 2009).

Our search identified 96 relevant papers, including 78 systematic and 18 non-systematically sourced studies, including a mix of experimental/observational, review and theoreti$\mathrm{cal} /$ modelling studies (Fig. 2). See the Supporting information for information for publication trend and bibliometric analysis, as well as summary tables of the traits/interaction types considered in each study and the effects on cooperation-antagonism. The following section provides a narrative overview and synthesis of these papers, where we describe major themes and patterns across empirical and theoretical studies from an ITV perspective. This includes: the types of interaction continua involved; traits related to outcome variance across all types of interaction; and mechanisms as well as constraints on shifts in interaction outcomes.

\section{Movement along cooperation-antagonism continua}

\section{Types and scales of continua}

Studies in our collection considered interaction types ranging from interspecific plant-animal and host-symbiont interactions, to intraspecific social and parent-offspring relationships (Fig. 3). Interactions between species can involve some exchange of resources and/or services (i.e. consumerresource interactions, Holland et al. 2005), where the actual costs and benefits of an interaction depend on the distribution and control of and competition for mutual resources, producing a dynamic landscape of mutualistic-antagonistic interactions (Jones et al. 2012). Outcome variation is found in plant-animal interactions, closely linked to the level of mutualistic services (e.g. pollination, seed dispersal, protection) or antagonistic effects from animal partners (e.g. destructive or exploitative feeding; Pesendorfer et al. 2016, RodriguezRodriguez et al. 2017). Plant-plant interactions may also vary from inhibitory to facilitative (Allen et al. 2018), and animal-animal interactions may be competitive or cooperative in relation to food resources (Perez-Barberia et al. 2015, Cantor et al. 2018) or other shared resources (e.g. nesting sites, Menzel and Blüthgen 2010). Host-symbiont interactions are a particular form of interspecific interaction where the balance of benefits, e.g. symbiont-conferred fitness gain, and costs, e.g. virulence, may determine outcomes. This may include animal/fungal/bacterial endo- and ectosymbionts 


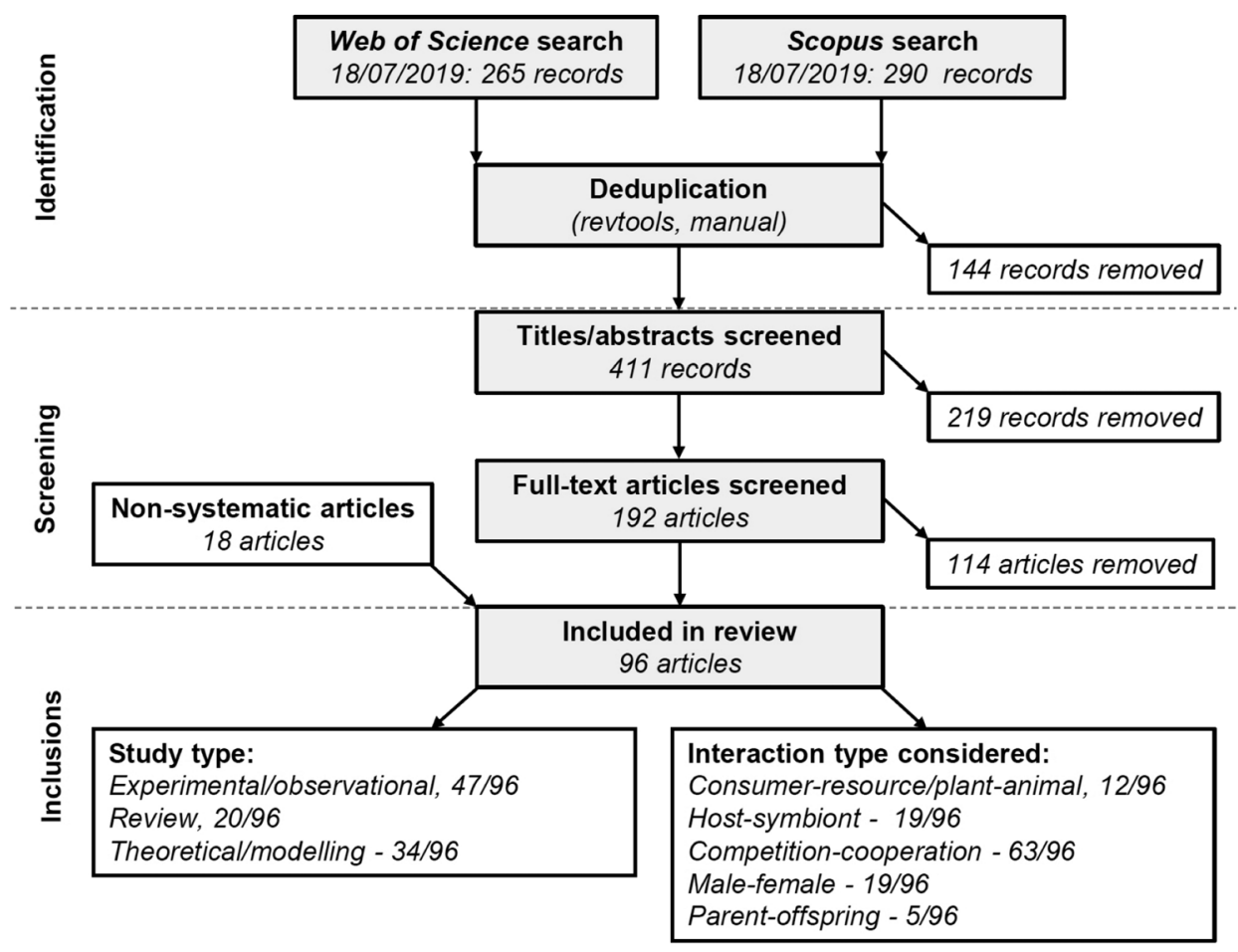

Figure 2. PRISMA (preferred reporting items for systematic reviews and meta-analyses) diagram of included studies. Summary information (study type and interaction type) for the studies that met our criteria is also included (note, the sum of studies in the groupings are $>96$ as several studies fall into multiple categories).

(Brown et al. 2012, Vorburger et al. 2013, Hajek et al. 2019), or physically independent symbionts (e.g. cleaner-client fish; Bshary and Grutter 2002). Note that there are also types of continua that are not represented in our data set although our search strategy was designed to find them. For example, we did not find studies on the continuum between predator-prey and mutualistic relationships in animal-animal interactions.

Qualitative outcome variation also appears in multiple intraspecific contexts, e.g. in social groups or dyads and between parents and offspring. Socially interacting animals may display cooperation and competition over foraging (Cords and Thurnheer 1993) or breeding (Marshall etal.2016). Cooperation in female-male interactions is often centered around parental care (e.g. brood care, nest defence in birds, Schuppe et al. 2016), and imbalances in care provision or partner desertion can represent antagonistic outcomes. A parent's decision not to contribute to parental care is also antagonistic towards offspring where it is detrimental to offspring development (although this is not always the case, Székely et al. 2006). The parent-offspring continuum may additionally extend to cooperatively breeding groups, where non-parent adults may provide group defence or direct care for offspring while competition or even conflict may also occur between adults and other group member's offspring (Trillmich 2010, Marshall et al. 2016).

There are key differences and similarities between interand intraspecific interaction scales. Cooperative outcomes may be more likely to evolve in intra- rather than interspecific interactions because of relatedness (i.e. through inclusive fitness), but competition may also be more intense between conspecifics than between heterospecifics due to the greater phenotypic similarity and resource requirements of participants (Barker et al. 2017). Nonetheless, interactions at each scale can produce mutual fitness benefits and thus cooperative outcomes with key mechanistic similarities. For example, asymmetries in resource use and production between parties influence the likelihood and benefits of inter- and intraspecific cooperation alike (Barker et al. 2017), and variation in resource use and specialization is common within species at magnitudes that may, in cases, be comparable to interspecific differences (Bolnick et al. 2003). Additionally, shifts toward cooperation at all hierarchical scales require that the direct and indirect fitness benefits of cooperation be balanced against the interests of individual units of selection, as highlighted in theoretical studies on evolutionary transitions from single- to multicellular organisms, where a unit may be an individual cell or an individual organism (Michod 1997). So despite most modelling studies focusing on intraspecific interactions (see publication trend analysis in the Supporting information), the mechanisms and processes they highlight are expected to have some mutual relevance across scales.

\section{Covariance of traits and interaction outcomes}

There are many empirical examples of apparent covariation between traits and outcomes of interspecific interactions, where the sex, age or individual genotype or phenotype of 
Interspecific-

I. Consumer/resource or plant/animal mutualism-antagonism continuum:

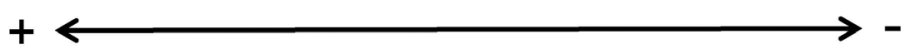

mutualism

antagonism

Types of interacting organisms: Any two species with a degree of control over a shared resource, which may include one or both species

Examples of relevant ITV: foraging tactics or preferences, competitor avoidance behaviour; pollination rewards and seed production rates

II. Host/symbiont mutualism-parasitism continuum:

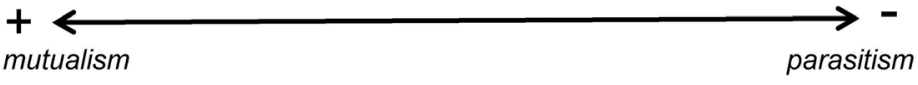

Types of interacting organisms: host species and their endosymbiotic or ectosymbiotic partner species

Examples of relevant ITV: symbiont dependence, protective effects against pathogens/predators/parasites, exploiting or cheating behavioural phenotypes
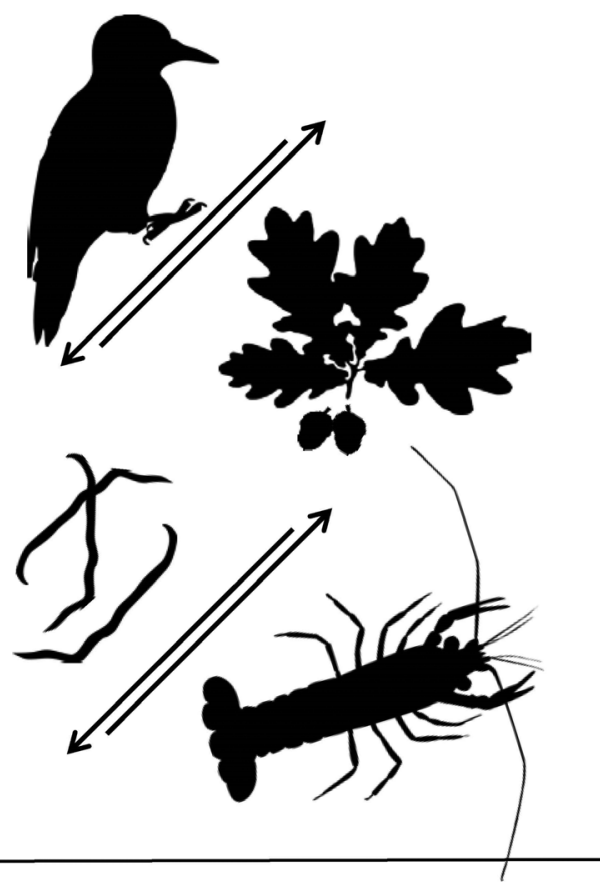

Intraspecific-

III. Conspecific cooperation-competition continuum:

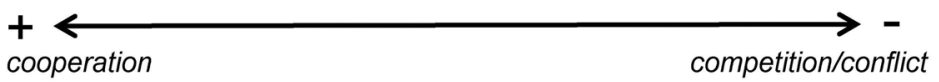

Types of interacting organisms: related or unrelated conspecifics in socially interacting dyads or networks; cooperative breeding groups

Examples of relevant ITV : competitive and cooperative behavioural phenotype, resource use, hormonal variation

IV. Female/male cooperation-conflict continuum:

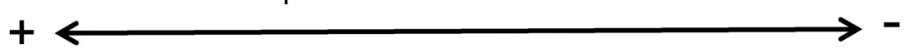
cooperation competition/conflict

Types of interacting organisms: male and female partners in a breeding pair

Examples of relevant ITV : behavioural phenotype, individual condition or attractiveness, reproductive potential

V. Parent/offspring care-conflict continuum:
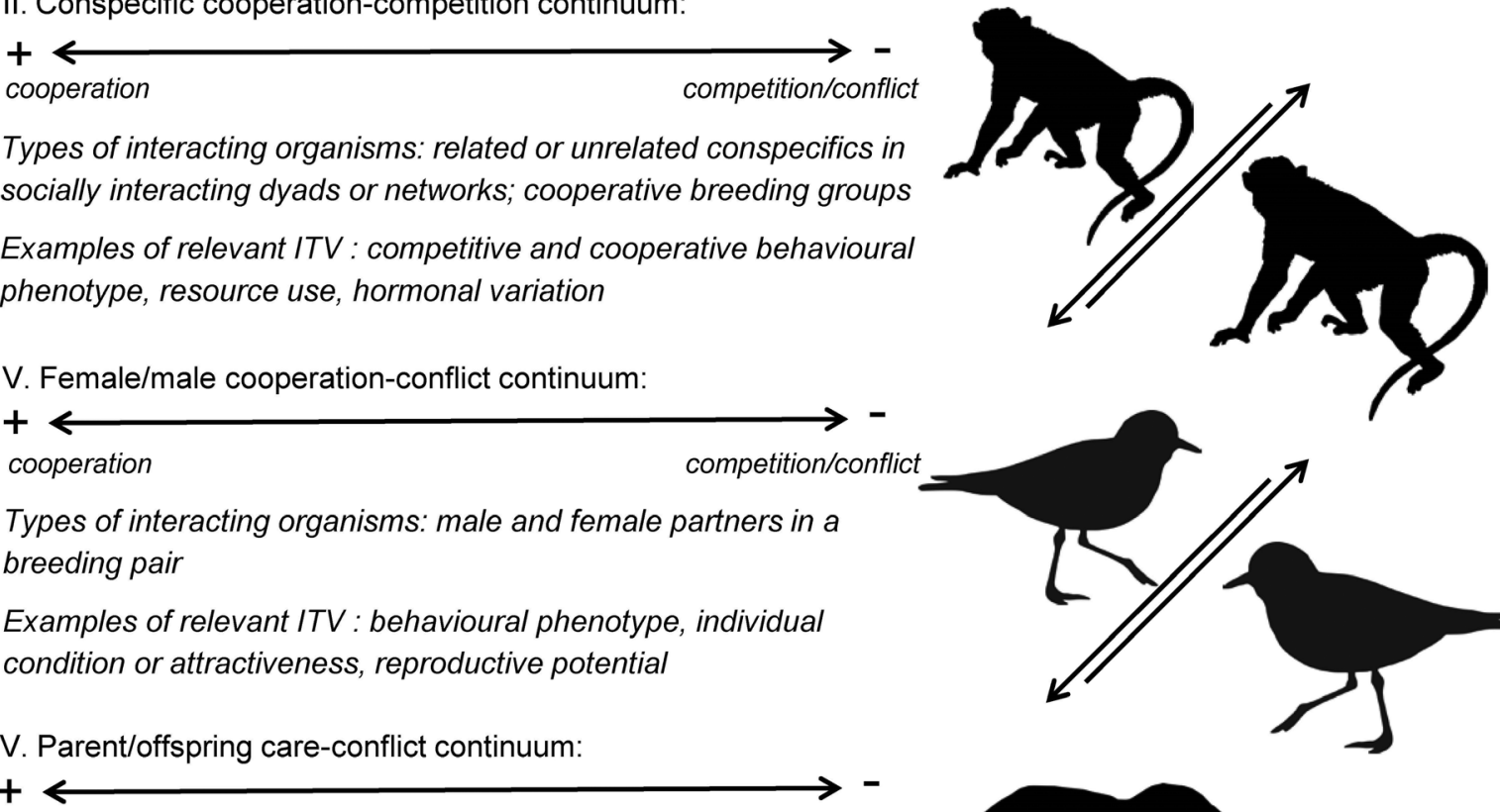

parental care

Types of interacting organisms: parents and offspring in species with parental care behaviour

Examples of relevant ITV : behavioural phenotype, individual condition, reproductive potential
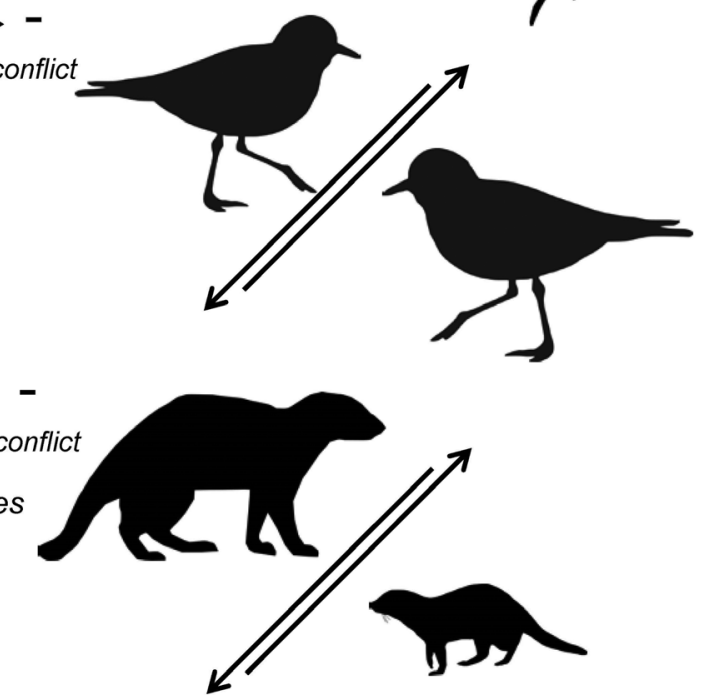

Figure 3. Conceptual diagram showing interaction types and continua focused on in this review at both the interspecific and intraspecific level, the types of organisms that constitute the parties to each interaction type, and some examples of traits that influence the quality of interaction outcomes. (Note, a small number of studies also consider variation along the cooperation-competition continuum for interspecific interactions.) 
either interaction participant may be linked to outcome variation (Thompson 1988). Importantly, this covariation may drive outcome variation via trait frequency effects (Fig. 1A). Examples of trait-outcome covariance include morphologi$\mathrm{cal}$ and reproductive traits in plants (e.g. flower and seed productivity; Cariveau et al. 2004, Pesendorfer et al. 2016), which can alter the relative strength and net quality of their interactions with animals, due to differences in foraging behaviour and preferences of mutualistic and antagonistic partner species. Behavioural traits are often related to the outcomes of interactions involving animals; for example bottlenose dolphins Tursiops truncatus display divergent foraging tactics where individuals either cooperate or directly compete with human fishermen (Cantor et al. 2018). This is a learned cooperative behaviour that reduces interspecific competition and investment in food searching, at the potential cost of higher intraspecific competition. Outcome variation has also been described for complex superorganisms; for example, colonies of nest-sharing ants Crematogaster modiglianii and Camponotus rufifemur may form shared nests or form single species nests (Menzel and Blüthgen 2010). When colonies form cooperative shared nests, benefits include common nest defence, shared foraging and brood care. In contrast, when one species (in particular C. modiglianii) forms separate nests, the species may compete over resources without providing these mutualistic benefits (i.e. a more antagonistic outcome). The outcome of their interactions is linked to the behavioural phenotypes of the colonies, including their tendency to initiate a nest, foraging behaviour, trail following and nest defence (Menzel and Blüthgen 2010).

Outcomes in host-symbiont interactions may be linked to genotypic variation (e.g. oogenesis-dependence on Wolbachia in parasitoid wasps, Dedeine et al. 2005; chemical defence traits in endophytic fungi strains, Yuan et al. 2011), and ontogenic variation (e.g. host sex and life-stage dependent effects of Poecilochirus carabi mites, De Gasperin and Kilner 2015). Also, either host or symbiont trait variation may influence outcomes, as in the symbiosis between crayfish (Cambarus spp.) and gill-cleaning ectosymbiotic branchiobdellidans (Cambarincola spp.). The interaction may be mutualistic, commensal or parasitic depending on the age/size of host, as well as variation in the feeding behaviour of symbionts that can cause gill scarring (Thomas et al. 2016).

For socially interacting conspecific animals, cooperationantagonism is often associated with behavioural phenotypes (e.g. participation in group foraging, offspring provisioning, territorial defence, intraspecific aggression). Amongindividual differences in cooperative-antagonistic behavioural traits may be consistent over time and underpinned by genetic or epigenetic variation, or due to state-dependent phenotypic plasticity (Helms Cahan 2001, Komdeur 2006, Sinervo et al. 2007, McAuliffe et al. 2015). State factors that can drive the expression in cooperative-antagonistic behaviours include body size (Whiteman and Côté 2004), physiology (Schoepf and Schradin 2013, Platt et al. 2016), learning and cognition (Platt et al. 2016), parasite infection status (Barber et al.
2017) and the social state of individuals (Roberts 1998, Hamilton and Ligocki 2012, Riebli et al. 2012, Singh and Boomsma 2015, Platt et al. 2016). For example, seed-harvester ant Messor pergandei foundresses may form cooperative multi-female nests or monogynous colonies, as determined by their aggressive-sociable phenotype, where each ant's response is determined by a combination of genetics and phenotypic plasticity in response to other females (Helms Cahan 2001, Helms and Helms Cahan 2012).

Behaviour is also key to the outcome of female-male and parent-offspring interactions, where the expression of cooperative behaviours often depends on an individual's state (e.g. condition-dependent turn taking in nest care/food provisioning in common murre, Uria aalge, Takahashi et al. 2017). An individual's future fitness expectations (i.e. their residual reproductive value) appears to be a key factor, where higher attractiveness, mate-finding ability and physical condition may lead to lower parental care investment and a higher likelihood of desertion (e.g. in male penduline tits Remiz pendulinus, van Dijk et al. 2010, 2012; and female Kentish plover, Charadrius alexandrinus, Székely et al. 2006). Few studies considered variation in other behaviours relating to femalemale interactions, e.g. mating behaviours. One exception is the pea leafminer Liriomyza huidobrensis, where males may use a highly cooperative dancing courtship strategy that improves female longevity and fecundity, or a more direct and possibly coercive strategy (Ge et al. 2019). Male courtship strategy is determined by their hunger state as female ovipositor punctures are also a food source. Patterns in intraspecific sexual conflict, reproductive skew and female choice across species (Bro-Jørgensen 2010, Surbeck et al. 2017) could provide a framework to also consider outcome variance in mating interactions.

Physiological (i.e. hormonal) mechanisms can play a role in intraspecific social and female-male interactions, which can be both state-dependent and show longer-term variation among individuals (Komdeur 2006, Trillmich 2010). For example, oxytocin levels affect decisions to behave cooperatively or competitively by influencing cognition in rhesus macaques (Macaca mulatta, Platt et al. 2016), highlighting that hormones may be important drivers shaping the individualised niche (Müller et al. 2020). However, levels of exogenous oxytocin in capuchins (Cebus apella, Smith et al. 2019) were unrelated to cooperative behaviour, suggesting that relationships between hormonal mechanisms and cooperationcompetition are species-specific. The reverse may also occur, with interaction outcomes influencing the hormonal state of individuals, as in the African striped mouse Rhabdomys pumilio. Here, males experimentally made to live a solitary as opposed to social reproductive lifestyle had reduced corticosterone and elevated testosterone levels compared to group-living mice (Schoepf and Schradin 2013). Hormone levels (e.g. prolactin, testosterone) can also be linked to the expression of parental care and intraspecific conflict behaviours (Schradin et al. 2009), highlighting potential feedbacks between physiological state and interactions. 


\section{Trait frequency effects}

This covariance between traits and interaction outcomes suggests that trait frequency effects (Fig. 1A) can drive net changes in interaction quality along cooperation-antagonism continua (Thompson 1988), for example through phenotypic plasticity in cooperation-antagonism linked traits. Plasticity in interspecific mutualistic-antagonistic behaviour may be driven by local environmental factors, such as population densities (e.g. attraction-repulsion behaviour in grazing herbivores, Perez-Barberia et al. 2015). In crayfish-branchiobdellida cleaning symbioses, damaging host-tissue feeding behaviour is promoted by high symbiont densities and food limitation (Brown et al. 2012, Thomas et al. 2016), highlighting resource use and availability as potential factors driving plasticity in mutualistic-antagonistic behaviours.

Interactions with third-party species can also be an environmental factor that indirectly shifts interspecific interactions between mutualism and antagonism. In the cooperative nest sharing of $C$. modiglianii and $C$. ruffermur ants, hemiepiphytic plants play a crucial role in nest stabilisation and promoting cooperative behaviours (Menzel and Blüthgen 2010). This trait frequency effect is a form of conditional or context-dependent outcome driven by phenotypic behavioural plasticity (sensu Bronstein 1994). Note, however, that context-dependent outcomes mediated by a third-party may also arise without any changes in trait frequency, as found in some host-symbiont interactions (e.g. Sirex noctilio woodwasp - Amylostereum areolatum fungal symbiont interactions mediated by the mycophagous nematodes, Hajek et al. 2019; Acyrthosiphon pisum-Hamiltonella defensa bacterial symbiont interactions mediated by parasitoid wasps, Vorburger et al. 2013, Niepoth et al. 2018).

Also for intraspecific interactions, environmental factors may drive state-dependent plasticity in cooperative-competitive behaviour. For example, southern hairy-nosed wombats Lasiorhinus latifrons behave more cooperatively and more readily share warrens in physical environments with harder soils (Walker et al. 2007). As above, environmental factors may also include indirect third-party effects, where intraspecific cooperativeness may be influenced by parasite loads (Stopka and Johnson 2012, Barber et al. 2017), or predatorprey interactions (Speed and Franks 2014). Environmental effects on parental and alloparental care are also expected (Komdeur 2006, Trillmich 2010). For example, in banded mongoose groups Mungos mungo, male-biased survival in low rainfall conditions leads to older males participating more in helping behaviour (Marshall et al. 2016). Environmental effects of cooperation are also the subject of modelling studies, suggesting that an individual's resource acquisition ability and the levels of food availability/resource supply should determine one's contribution to cooperative foraging (Dubois and Giraldeau 2003, Liu et al. 2020). Specifically, individuals that are more capable of acquiring resources should be more likely generally to invest more in cooperative behaviours, but under harsher environmental conditions (i.e. reduced resources), the relative investment of subordinate individuals in cooperative behaviours increases (as was empirically demonstrated in burying beetles, Nicrophorus nepalensis, Liu et al. 2020).

Intrinsic state factors are also linked to variation in the expression of conspecific cooperation, another example of trait frequency effects driven by phenotypic plasticity. This includes ontogenetic variation (e.g. age-dependent coalition formation in Barbary macaque, Macaca sylvanus; Rathke et al. 2017), body condition (e.g. condition-dependent helper responsiveness in M. mungo; Bell 2008) and/or future fitness expectations (e.g. helping behaviour in wasps such as Polistes dominulus and Liostenogaster flavolineata, Field and Cant 2006). Group size may also be particularly important; for example, studies in non-human primates suggest that smaller sub-groups may be more likely to form as the total size of interacting conspecifics increases (Sih et al. 2009). Individuals then behave cooperatively with subgroup members, but there is a net increase in the proportion of competitive interactions across the larger network.

Although the majority of trait frequency effects found here were due to phenotypic plasticity in traits, natural selection may also change the proportion of cooperative-competitive traits within a population. For example, pine engraver Ips pini phenotypes differ in their host searching behaviour (i.e. pioneers versus responders, Aukema and Raffa 2004). Exploitative responding phenotypes appear more susceptible to predation, such that elevated predation pressure may increase the proportion of cooperative pioneer phenotypes within populations.

\section{The importance of partner identity}

The identity of one's interaction partner can determine the direct and indirect fitness benefits for an individual and their expression of cooperative-competitive behaviour. Partner identity can be involved in trait frequency effects (Fig. 1A), if it influences the expression of traits directly related to cooperation or antagonism, or in systematic variance effects (Fig. 1B) if variation among interaction partners alters the costs and benefits of the interaction. Particularly important for intraspecific interactions is the level of relatedness with a partner, and the potential inclusive-fitness effects. As an example, in the cnidarian Hydractinia symbiolongicarpus, where colony fusion is determined genetically: colonies sharing allorecognition alleles fuse, whereas colonies that do not will engage in conflict (Nicotra et al. 2009). Similarly, affiliative interactions in social yellow-bellied marmot groups Marmota flaviventer, appear to be structured around kin relationships between individuals, their age and their individual social state (Wey and Blumstein 2010, Wey et al. 2019).

On the one hand, the degree of relatedness between individuals can be seen as a biotic environmental factor that may cause one to behave more or less cooperatively, for example through kin selection (Hamilton 1964), as evidenced by empirical studies highlighting the importance of partner identity in cooperative decision-making (Nicotra et al. 2009, Wey et al. 2019). On the other hand, relatedness among individuals in a group or population is also related to the 
level of phenotypic variation or similarity within the group, as more related individuals tend to exhibit more similar traits. Modelling results have further highlighted that higher ingroup relatedness can increase the willingness of group members to contribute to offspring-rearing in collective breeding groups (Savage et al. 2013), or the likelihood with which individuals induce herd defences (Frank 1998). So here cooperation increases with increasing genetic similarity between individuals, due to inclusive fitness effects.

Cooperation between phenotypically similar, but not necessarily related, individuals may also result from social discrimination or tag-based cooperation, where individuals may cooperate based on sharing the same (potentially arbitrary) trait. Hochberg et al. (2003) provide a model showing that when social discrimination evolves, it may cause phenotypically similar individuals to form into different, spatially distinct groups. Similarly, interaction outcomes may depend on cultural traits. In a simple form, this may be an arbitrary trait that defines the cooperative social group ('in-group favoritism'), or provides the possessor with prestige ('prestige hierarchy', Ihara 2011).

In the cases described so far, individuals preferentially cooperate with related or otherwise similar individuals, but theoretical studies suggest that the opposite - cooperation with dissimilar individuals - may also be beneficial. For pairs or groups of interacting individuals, variation among the interacting participants can provide direct benefits, giving rise to cooperative/mutualistic outcomes. Such benefits have been termed 'social heterosis' (Nonacs and Kapheim 2007), and can be considered systemic variance effects. For example, Uitdehaag (2011) models a system where individuals differ in how their resource acquisition is affected by environmental fluctuations. This proposes that fitness can be buffered if individuals engage in a mutualism with partners that are different from them, so they perform well in different conditions and can support each other at different times. Such mutualisms would only emerge when there is sufficient intraspecific variation in environmental responses.

Beneficial effects of variation between participants and individual specialisation may occur in various types of interactions. In male-female interactions, intraspecific variation in reproductive roles is an inherent characteristic of the interaction partners. Barta (2016) shows that increased role/task specialisation (e.g. sexes providing distinct forms of parental care) can promote the evolution of cooperation in male-female interactions, and similarly, task specialisation can promote cooperation between conspecifics (e.g. the use of using different foraging strategies). Modelling of inter- or intraspecific microbial interactions suggests that the degree of functional specialisation may be non-linearly related to the qualitative outcome of interactions (Estrela et al. 2016), where intermediate levels of functional benefits provided between partners are most likely to lead to cooperative outcomes. Considering leadership hierarchies in social groups as a form of task/role specialisation, social hierarchies may make social groups more efficient but this must be counterbalanced by within-group conflict over leadership (Hamilton 2013,
Pinter-Wollman et al. 2016). These studies highlight that the formation of a cooperative or antagonistic interaction relies on balancing the benefits of cooperation with the interests of the individuals, where one's interaction partner and the potential benefits it may provide are key factors. In each of the cases above, where the outcome of an interaction is based on trait similarity (or dissimilarity) between partners, there is the potential for systemic variance effects to emerge, as changes in the level of ITV in a population will alter the degree of differences between potential interaction partners.

\section{Systemic variance effects}

System variance effects describe cases when a change in a population's level of ITV leads to outcome variation at the population level, even if there is no direct trait-outcome covariation. Notably, studies in our collection focusing on such variance effects were predominantly theoretical and focused on intraspecific social interactions. For example, theory suggests that the level of ITV in fecundity can alter patterns of relatedness within neighbourhoods, which can in turn produce outcome variation at the group level (Rodrigues and Gardner 2013).

Prime examples of systemic variance effects concern ITV in competitive ability. For example, cooperative food sharing can emerge with greater variation in resource acquisition between individuals (Uitdehaag 2011, Barta 2016). Contrastingly, in hierarchical populations, greater ITV in competitive foraging ability may reduce cooperative foraging as it becomes detrimental to lower-ranked individuals (Ranta et al. 1993). This is reflected in three-spined sticklebacks who prefer schooling with similar-sized individuals (Ranta et al. 1992). These findings suggest that phenotypic similarity in competitive ability can also promote cooperative outcomes within social groups, despite the common assumption that there is more competition between phenotypically similar individuals (Barker et al. 2017). The direction of the effect may also be context-dependent, for example in a producer-scrounger game, when resources are scarce, individuals become less likely to exploit food patches found by others as the variation in competitive ability increases, because only the most competitive individuals have a chance to scrounge the few encountered food patches (Lee et al. 2016). But if resources are plenty, scrounging frequency goes up with increasing ITV in competitive ability because more individuals have the chance to take advantage of a large number of encountered food patches.

In the competition for mating partners, if there is ITV in strength, costly displays can evolve that help resolve potential fights and thus reduce antagonistic interactions (McNamara and Leimar 2010). Again, this effect can also go in the other direction. Franz et al. (2011) find that in a model with nonheritable variation in fighting ability, increasing ability of interacting individuals to accurately assess who is strongest destabilizes cooperative turn-taking strategies. Thus, the interaction should become more antagonistic with increasing ITV in fighting ability. Furthermore, genetic variation in traits related to competitive ability such as body size can 
lead to indirect genetic effects. For example, Marjanovic et al. (2018) studied a model with intraspecific variation in body size where an individual's growth depended on both its own trait and the difference in trait compared to an interacting individual, multiplied by an interaction coefficient. The interaction coefficient could range from antagonistic (larger individuals behaved aggressively towards smaller individuals) to cooperative (larger individuals helped smaller individuals) and coevolved with trait variation. There is substantial evidence that the level of ITV in competitive ability can lead to outcome variation in intraspecific interactions, while the direction of the effects may be positive or negative and potentially context-dependent.

Similar to variation in competitive ability, the level of ITV in mate quality or attractiveness in a population may also drive outcome variation in female-male interactions. The more variation in male quality, the more repeatable is male mate finding success, and the less willing males may be to invest in raising individual broods (Lehtonen and Kokko 2012).

\section{The stabilisation of cooperation via systemic variance effects and partner control mechanisms}

In this subsection, we look at the effect of ITV in cooperativeantagonistic behaviours on the emergence, maintenance and breakdown of predominantly cooperative/mutualistic interactions. This can be considered a specific case where interaction outcomes are driven by both systemic variance effects and trait-specific selective pressures (i.e. trait frequency effects). In mutualisms, individuals may cheat and employ strategies that are detrimental to their interaction partner (e.g, feeding on host tissue in cleaner-client fish mutualisms, Bshary and Grutter 2002), where 'cheater' phenotypes may receive a fitness advantage by gaining benefits from the partner species without reciprocating (Frederickson 2013, Sachs 2013). While this may potentially lead to mutualism breakdown (for example a rapid shift towards parasitism in the jellyfish symbiont Symbiodinium microadriaticum; Sachs and Wilcox 2006), cases of evolutionary breakdowns appear relatively rare (Sachs and Simms 2006). Furthermore, control mechanisms against antagonistic individuals are often observed, for example through partner choice (Bshary and Shäffer 2002, Jones et al. 2012) or selective provisioning of benefits based on the partner behaviour (Edwards et al. 2006). Therefore, cheater phenotypes (introduced e.g. through increasing ITV in behaviour) are often subject to trait specific selective pressures intrinsic to their mutualistic partner (i.e. sanctions) that promote and maintain high levels of mutualistic phenotypes.

Cheating also occurs in predominantly cooperative intraspecific interactions. The emergence of cheater phenotypes represents shifts towards antagonism within populations but often occurs only at low frequencies because of negative frequency-dependent selection (Kraemer and Velicer 2014), or other costs associated with the cheating (Aukema and Raffa 2004). Control mechanisms may arise such as partner choice (Roberts 1998), or policing (i.e. aggressive behaviour towards conspecifics as a punishment for mediating fitness losses due to competition, Singh and Boomsma 2015). In social insects, for example, the more efficient policing is, the more cooperation (i.e. less selfish egg-laying by workers) is observed (Wenseleers and Ratnieks 2006). Models further suggest that cooperative behaviour can also be preserved through selfrestraint in highly related populations, without the need for policing (Frank 1996). The partner control mechanisms of a species or the composition of policing phenotypes within a population are factors that can buffer against the introduction of more antagonistic individuals into a cooperative society and increase the overall level of cooperation.

ITV may be essential to the evolution of partner control mechanisms and the stability of primarily mutualistic/cooperative interactions even in the face of cheaters. Variation in partner quality introduced through various mechanisms (e.g. mutation, McNamara et al. 2004; individual errors in decision making; Ito et al. 2017; migration, Foster and Kokko 2006) can ensure that there are always sufficiently many cheater/defector phenotypes to incentivise the evolution and maintenance of partner control mechanisms in cooperative social groups (McNamara and Leimar 2010) and interspecific mutualisms (Johnstone and Bshary 2008, Song and Feldman 2013). For example, Foster and Kokko (2006) studied a host-symbiont model and found that sufficient standing genetic variation in symbiont quality is required to maintain host choice and stabilise the mutualism. Analogous to cheater phenotypes, the emergence of antagonistic defector cells (e.g. through mutation) and control mechanisms appear to stabilise higher-level units of organisation in transitions to multicellularity (Michod and Roze 2001, Michod and Nedelcu 2003, Rainey and Kerr 2010). In this case, first, an increase in ITV supplies more cheater/defector individuals without necessarily shifting the average interaction outcome (systemic variance effect), and this then induces selection pressures that change the mean traits (e.g. host choosiness, and then by consequence also symbiont quality) leading to a shift in the net interaction outcome towards greater cooperation (trait frequency effect, Fig. 1C, pathway ii).

\section{Discussion}

For a long time, ITV was considered as noise around an optimum value, but variation both between and within individuals is increasingly recognised as an important factor influencing various ecological processes (Bolnick et al. 2003, 2011, Dall et al. 2012, Violle et al. 2012, Westneat et al. 2015). In this review, we identified two mechanisms through which ITV influences outcome variation along cooperative-antagonistic continua. First, where there is trait-outcome covariance in inter- or intraspecific interactions (Sinervo et al. 2007, Cantor et al. 2018), changes in the frequency of those traits may alter the quality of the average interaction across a population (termed trait frequency effects, Fig. 1A). While many processes can lead to frequency changes, we identified empirical examples of trait-specific selective pressures due to extrinsic or intrinsic state factors (Aukema and Raffa 2004, 
Sinervo et al. 2007, Jones et al. 2012), and phenotypic plasticity (Walker et al. 2007, Menzel and Blüthgen 2010, van Dijk et al. 2010, 2012, Marshall et al. 2016). Also, geographic variation in traits (Helms and Helms Cahan 2012) suggests that demographic changes through migration may be a source of outcome variation. It has long been recognised that interaction outcomes may be conditional or context-dependent, i.e. where the ecological context of an interaction, such as physical conditions or third-party species alter the costs and benefits for interaction partners (Bronstein 1994, Chamberlain et al. 2014; see for example Hajek et al. 2019). While contextdependency may also occur without changes in trait frequency, e.g. through changing the frequencies of certain interactions or their payoffs (cases that are often outside the scope of our study because they are not driven by ITV), our systematic review identified many examples of outcome variance associated with behavioural plasticity. Behavioural traits may also be subject to selection (Smith and Blumstein 2008, Trillmich et al. 2018), highlighting the particular importance of behavioural traits to context-dependent outcomes.

Second, we described systemic variance effects (as in Fig. 1B), where the level of ITV in a trait in a population (as opposed to the mean value) is the factor that influences the cooperative-antagonistic outcome. As in social discrimination and kin selection contexts (Hamilton 1964, Hochberg et al. 2003), greater among-individual variation may be expected to decrease cooperation with conspecifics due to reduced indirect fitness benefits. In these cases, the outcome variance appears to manifest through state-dependent expression of cooperative-competitive behaviour at the individual level (e.g. as in Rodrigues and Gardner 2013, where the average individual would behave less cooperatively in a high ITV population). Therefore, a systemic variance effect derived from variance in one trait may occur by inducing plasticity or selection in another trait (i.e. induce a trait frequency effect, Fig. 1C). While these effects are closely entwined and outcome variation in a system may be driven by a combination of both (e.g. in the case of partner control mechanisms), we consider it to be important to distinguish between mean trait and variance effects, as different individual-level processes can drive changes in the mean versus variance of traits within populations.

While processes like selection and phenotypic plasticity can shift the mean trait value within a population, the level of ITV in a population is also not constant and may be influenced by multiple processes. For example, mutation and migration/invasion may be sources of ITV and introduce rare antagonistic phenotypes in predominantly mutualistic partner species (as suggested by Foster and Kokko 2006, Frederickson 2013). Furthermore, selective pressure can influence levels of ITV either directly, through selection on traits linked to interaction quality, or indirectly through evolutionary tradeoffs (Wolf and Weissing 2010). Additionally, phenotypic plasticity may also be a source of ITV where there is environmental variation at an individual level, and/or where individuals vary in their response to the environment (e.g. reaction norms, Nussey et al. 2007, Dingemanse et al.
2010), such as trait canalization under optimal condition (Careau et al. 2014), or exposure of cryptic genetic variation under extreme conditions (Paaby and Rockman 2014). Environmental effects can alter levels of both between- and within-individual variation, through bet-hedging strategies or sub-optimal performance (Wolf and Weissing 2010). Thus, many factors influence the degree of ITV within a population and how stable that variation is over time, which will have implications for the stability of interaction outcomes.

We expect that the level of ITV and the type of trait involved in outcome variance is likely to influence the nature of cooperative-antagonistic shifts and the time scale on which they happen. Both systemic variance effects and trait frequency effects can occur on the time scale of an individual life span if the traits involved show state-dependent phenotypic plasticity, or on a longer evolutionary time scale spanning multiple generations if ITV is linked to heritable genetic variation. For example, plasticity in behavioural phenotypes may occur rapidly (as in Perez-Barberia et al. 2015), but behavioural traits can also be highly consistent over time and heritable (Bergmüller et al. 2010) [e.g. dolphin foraging behaviours (Cantor et al. 2018), which may be maternally transmitted to offspring (Whitehead 2017)]. Moreover, expressing phenotypic plasticity and maintaining the capacity to do so (e.g. via cognitive capacities) may involve costs, which impose limits on plastic responses (Dewitt and Scheiner 2004). Furthermore, the degree to which ITV is linked to heritable genetic variation may determine the long term consequences of trait frequency effects. Where traits that determine interaction outcomes are closely associated with an allele (Nicotra et al. 2009), changes in allele frequency in the population will have intergenerational effects in interaction outcomes. In extreme cases, selection on heritable traits can lead to qualitative evolutionary consequences, e.g. mutualism breakdown, although this appears rare potentially due to the influence of partner control mechanisms (Frederickson 2013, Sachs 2013). Whether any shift occurs, the persistence of that shift, and its long-term implications may therefore be influenced by the ITV involved, where highly labile traits linked to interaction outcomes may be associated with shifts over short time scales, and more stable or heritable traits may be associated with longer-term shifts with evolutionary consequences.

In our narrative overview of papers, some themes were prominent in both empirical and theoretical studies, for example the degree of relatedness between participants. Additionally, theory suggests that ITV in mutualist quality is required to maintain host-symbiont mutualisms, because this maintains selection pressures for partner choice (Song and Feldman 2013), where partner choice in response to mutualist variation is supported by empirical evidence (Bshary and Schäffer 2002). On the other hand, studies considering the role of ITV and partner controls in maintaining stable cooperative parent-offspring and female-male interactions were largely absent. It is also notable that although many of theoretical papers in our collection remain abstract, some of the 
theoretical papers are inspired by, or linked with, the behaviour of specific species (Sinervo et al. 2007 on lizards, van Dijk et al. 2012 on penduline tits).

There were also clear differences in topics covered in empirical and theoretical publications in our collection. Among empirical studies, we found comparable numbers of papers for intraspecific versus interspecific interactions, but the vast majority of the theoretical papers in our data set concern intraspecific interactions (see publication trend analysis including comparative proportions of interaction scales by study type in the Supporting information). An interesting mechanism among the empirical papers that did not appear in any of the theoretical papers includes shifts between interspecific competition and cooperation (e.g. dolphins and fishermen, ants). Another striking difference was that many theoretical papers but only few empirical papers represent systemic variance effects at the population level (Fig. 1B). For example, some of the theoretical papers suggest that trait differences can serve as a tag for social discrimination and thereby promote cooperation or provide information on competitiveness and fighting ability (Hochberg et al. 2003, McNamara and Leimar 2010). Some of the theoretical papers also concern direct benefits of variation among interacting individuals (Nonacs and Kapheim 2007, Uitdehaag 2011), a mechanism that did not appear among empirical studies. Many empirical papers, on the other hand, focus on ITV's role in determining whether the outcome of an interaction, for example between an individual plant and its associated animal species, is antagonistic or cooperative. Such trait shifts at the individual level may then lead to trait frequency effects.
Are the differences in literature coverage biologically meaningful, do they represent differences in the amount of attention given to certain phenomena, or are they potentially a result of our search/screening pipeline (e.g. due to the choice of search terms, inclusion criteria etc.)? We speculate that practical considerations are a major factor, e.g. the shortage of empirical papers reporting systemic variance effects may exist because of the additional burden of conducting experiments where treatments manipulate population ITV, requiring replication at the population level. Similarly, experimentally testing the effect of partner control mechanisms in maintaining the evolutionary stability of mutualisms likely requires relatively longer-term, multi-generation studies, while modelling approaches are well suited to dealing with evolutionary timescales. Based on the differences between empirical research and theory, we suggest that the following topics may be interesting areas for future research. Although logistically challenging, future experiments that manipulate the level of ITV and track shifts at the population level and/or at an evolutionary time scale (e.g. in experimental evolution studies with short-lived organisms) would be extremely valuable. Such experiments could test predictions by some of the theoretical papers in our data set, for example whether variation in fecundity among individuals promotes cooperation by increasing relatedness (Rodrigues and Gardner 2013), or further explore the directional conditionality of systemic variance effects due to ITV in competitive ability (picking up from Ranta et al. 1992, 1993). On the theoretical side, we suggest that it would be valuable to build models that allow shifts along the antagonism-cooperation

\section{Glossary}

Trait. Any characteristic of an individual organism that may or may not be heritable, including genotype, sex, morphology, behaviour, condition, social status etc.

Intraspecific trait variation 'ITV'. Variation in traits among individuals of the same species, (i.e. among-individual variation), including variation driven by phenotypic plasticity (i.e. within-individual variation; per Dingemanse et al. 2010).

Phenotypic plasticity. Phenotypic expression influenced by intrinsic or extrinsic environmental factors, including an individual's current environment (i.e. state-dependent plasticity, per Wolf and Weissing 2010), or their past/early-life environment (e.g. developmental plasticity; Dewitt and Scheiner 2004).

Scale of an interaction. The hierarchical level under consideration, e.g. cells within an individual, individuals within a population or multiple interacting species.

State. Any element of an individual's extrinsic or intrinsic environment that is strategically relevant to their future fitness (Wolf and Weissing 2010).

Interaction quality. The outcome of an interaction along a cooperative-antagonistic continuum.

Outcome variance. Variation in interaction quality, e.g. where interaction quality differs between individuals of the same population, or the interaction quality for an individual or a population changes over time or context.

Trait frequency effect. Changes in the frequency of a trait in a population leading to a net change in interaction quality. This may apply to interactions within the population, or interactions of that population with another species or conspecific group.

Systemic variance effect. Changes in the level of ITV within a population leading to a net change in interaction quality, e.g. an interaction becomes more or less cooperative/antagonistic due to an increase or decrease in trait variation within a population. 
axis to emerge more mechanistically from the underlying biological processes, taking into account costs and benefits at the individual level. For example, models for plant-animal interactions could take into account multiple processes (e.g. herbivory and pollination) and study how intraspecific variation in one or both partners affects the quality of the interaction.

We believe there is value in considering shifts on cooperation-antagonism continua from an individual perspective, as phenotypic variation among individuals is a key component of mechanisms that lead to shifts in the qualitative outcomes of interactions in ecological timeframes. There is a diverse range of traits involved (although behavioural ITV appears particularly important), wherein the level of ITV and the particular trait involved (particularly its plasticity and heritability) are likely to influence interaction-outcome variance over ecological time frames, and their long-term evolutionary implications. Notably, the research interest in this topic is greatly increasing across interaction scales and interaction types, and there is wide scope for future integration given the particularly broad scope of this topic (for further information see publication trend and bibliometric analysis in the Supporting information). We also believe that applying current evidence synthesis techniques as we have here (i.e. systematic literature searches, transparently reported and reproducible review methods etc.) can promote greater integration of empirical research and theory into this topic to better inform future research.

Acknowledgement - Thank you Niki Teunissen for assisting with database searches.

Funding - This project has received funding from the European Union's Horizon 2020 research and innovation programme under the Marie Sklodowska-Curie grant agreement no. 836937 (NPM). The Centre for Ocean Life is a VKR center of excellence supported by the Villum foundation. This research was funded by the German Research Foundation (DFG) as part of the SFB TRR $212\left(\mathrm{NC}^{3}\right.$; project no. - 316099922, 396777092, 396777467, 396777869, 396779914, 396780003, 396780988, 396782445, 396782288, 396782608).

\section{Author contributions}

Note, other than NPM and MJW, the author list is in alphabetical order. Nicholas P. Moran: Conceptualization (equal); Data curation (lead); Formal analysis (lead); Funding acquisition (equal); Investigation (equal); Methodology (lead); Project administration (equal); Software (lead); Validation (equal); Visualization (lead); Writing - original draft (equal); Writing - review and editing (lead). Barbara A. Caspers: Conceptualization (equal); Funding acquisition (equal); Investigation (equal); Writing - original draft (equal); Writing - review and editing (supporting). Nayden Chakarov: Conceptualization (equal); Investigation (equal); Writing original draft (equal); Writing - review and editing (supporting). Ulrich R. Ernst: Conceptualization (equal); Investigation (equal); Writing - original draft (equal); Writing - review and editing (supporting). Claudia Fricke: Conceptualization (equal); Funding acquisition (equal); Investigation (equal); Writing - original draft (equal); Writing - review and editing (supporting). Joachim Kurtz: Conceptualization (equal); Funding acquisition (equal); Investigation (equal); Writing - original draft (equal); Writing - review and editing (supporting). Navina D. Lilie: Conceptualization (equal); Investigation (equal); Writing - original draft (equal); Writing - review and editing (supporting). Lai Ka Lo: Conceptualization (equal); Investigation (equal); Writing original draft (equal); Writing - review and editing (supporting). Caroline Müller: Conceptualization (equal); Funding acquisition (equal); Investigation (equal); Writing - original draft (equal); Writing - review and editing (supporting). Reshma R: Conceptualization (equal); Investigation (equal); Writing - original draft (equal); Writing - review and editing (supporting). Elina Takola: Conceptualization (equal); Data curation (supporting); Formal analysis (supporting); Investigation (equal); Software (supporting); Validation (equal); Visualization (supporting); Writing - original draft (equal); Writing - review and editing (supporting). Pete C. Trimmer: Conceptualization (equal); Investigation (equal); Writing - original draft (equal); Writing - review and editing (supporting). Koen J. van Benthem: Conceptualization (equal); Investigation (equal); Writing - original draft (equal); Writing - review and editing (supporting). Jamie Winternitz: Conceptualization (equal); Investigation (equal); Writing - original draft (equal); Writing - review and editing (supporting). Meike J. Wittmann: Conceptualization (equal); Funding acquisition (equal); Investigation (equal); Methodology (supporting); Project administration (equal); Supervision (lead); Writing - original draft (equal); Writing review and editing (lead).

\section{Data availability statement}

Data and code used to process our systematic searches, screening records, and the information extracted from studies that was used to produce all tables and figures presented here are all available through Open Science Framework; https://osf. io/9kfpc/ (doi: 10.17605/OSF.IO/9KFPC).

\section{References}

Allen, W. J. et al. 2018. Intraspecific variation in indirect plant-soil feedbacks influences a wetland plant invasion. - Ecology 99: 1430-1440.

Arnqvist, G. and Rowe, L. 2005. Sexual conflict. - Princeton Univ. Press.

Aukema, B. H. and Raffa, K. F. 2004. Gender- and sequencedependent predation within group colonizers of defended plants: a constraint on cheating among bark beetles? - Oecologia 138: 253-258.

Barber, I. et al. 2017. Parasitism, personality and cognition in fish. - Behav. Process. 141: 205-219.

Barker, J. L. et al. 2017. Synthesizing perspectives on the evolution of cooperation within and between species. - Evolution 71: 814-825. 
Barta, Z. 2016. Individual variation behind the evolution of cooperation. - Phil. Trans. R. Soc. B 371: 20150087.

Bell, M. B. 2008. Strategic adjustment of begging effort by banded mongoose pups. - Proc. R. Soc. B 275: 1313-1319.

Bergmüller, R. et al. 2010. Evolutionary causes and consequences of consistent individual variation in cooperative behaviour. Phil. Trans. R. Soc. B 365: 2751-2764.

Bolnick, D. I. et al. 2003. The ecology of individuals: incidence and implications of individual specialization. - Am. Nat. 161: 1-28.

Bolnick, D. I. et al. 2011. Why intraspecific trait variation matters in community ecology. - Trends Ecol. Evol. 26: 183-192.

Bro-Jørgensen, J. 2010. Intra- and intersexual conflicts and cooperation in the evolution of mating strategies: lessons learnt from ungulates. - Evol. Biol. 38: 28-41.

Bronstein, J. L. 1994. Conditional outcomes in mutualistic interactions. - Trends Ecol. Evol. 9: 214-217.

Brown, B. L. et al. 2012. The fine line between mutualism and parasitism: complex effects in a cleaning symbiosis demonstrated by multiple field experiments. - Oecologia 170: 199-207.

Bshary, R. and Grutter, A. S. 2002. Experimental evidence that partner choice is a driving force in the payoff distribution among cooperators or mutualists: the cleaner fish case. - Ecol. Lett. 5: 130-136.

Bshary, R. and Schäffer, D. 2002. Choosy reef fish select cleaner fish that provide high-quality service. - Anim. Behav. 63: 557-564.

Canestrari, D. et al. 2014. From parasitism to mutualism: unexpected interactions between a cuckoo and its host. - Science 343: 1350-1352.

Cantor, M. et al. 2018. Spatial consequences for dolphins specialized in foraging with fishermen. - Anim. Behav. 139: 19-27.

Careau, V. et al. 2014. Early-developmental stress, repeatability and canalization in a suite of physiological and behavioral traits in female zebra finches. - Integr. Comp. Biol. 54: 539-554.

Cariveau, D. et al. 2004. Direct and indirect effects of pollinators and seed predators to selection on plant and floral traits. Oikos 104: 15-26.

Chamberlain, S. A. et al. 2014. How context dependent are species interactions? - Ecol. Lett. 17: 881-890.

Cords, M. and Thurnheer, S. 1993. Reconciling with valuable partners by long-tailed macaques. - Ethology 93: 315-325.

Dall, S. R. X. et al. 2012. An evolutionary ecology of individual differences. - Ecol. Lett. 15: 1189-1198.

De Gasperin, O. and Kilner, R. M. 2015. Friend or foe: interspecific interactions and conflicts of interest within the family. - Ecol. Entomol. 40: 787-795.

Dedeine, F. et al. 2005. Wolbachia requirement for oogenesis: occurrence within the genus Asobara (Hymenoptera, Braconidae) and evidence for intraspecific variation in A. tabida. Heredity 95: 394-400.

Des Roches, S. et al. 2018. The ecological importance of intraspecific variation. - Nat. Ecol. Evol. 2: 57-64.

Dewitt, T. and Scheiner, S. 2004. Phenotypic plasticity: functional and conceptual approaches. - Oxford. Univ. Press.

Dingemanse, N. J. et al. 2010. Behavioural reaction norms: animal personality meets individual plasticity. - Trends Ecol. Evol. 25: 81-89.

Dubois, F. and Giraldeau, L. A. 2003. The forager's dilemma: food sharing and food defense as risk-sensitive foraging options. Am. Nat. 162: 768-779.
Edwards, D. P. et al. 2006. Selection for protection in an ant-plant mutualism: host sanctions, host modularity and the principalagent game. - Proc. R. Soc. B 273: 595-602.

Estrela, S. et al. 2016. Private benefits and metabolic conflicts shape the emergence of microbial interdependencies. - Environ. Microbiol. 18: 1415-27.

Field, J. and Cant, M. A. 2006. Helping effort in primitively eusocial wasps. - Ann. Zool. Fenn. 43: 481-487.

Foster, K. R. and Kokko, H. 2006. Cheating can stabilize cooperation in mutualisms. - Proc. R. Soc. B 273: 2233-2239.

Frank, S. A. 1996. Policing and group cohesion when resources vary. - Anim. Behav. 52: 1163-1169.

Frank, S. A. 1998. Inducible defence and the social evolution of herd immunity. - Proc. R. Soc. B 265: 1911-1913.

Franz, M. et al. 2011. The evolution of cooperative turn-taking in animal conflict. - BMC Evol. Biol. 11: 323.

Frederickson, M. E. 2013. Rethinking mutualism stability: cheaters and the evolution of sanctions. - Q. Rev. Biol. 88: 269-295.

Ge, J. et al. 2019. Sexual cooperation relies on food controlled by females in agromyzid flies. - Anim. Behav. 149: 55-63.

Hajek, A. E. et al. 2019. Context-dependent interactions of insects and defensive symbionts: insights from a novel system in siricid woodwasps. - Curr. Opin. Insect Sci. 33: 77-83.

Hamilton, I. M. 2013. The effects of behavioral plasticity and leadership on the predictions of optimal skew models. - Behav. Ecol. 24: 444-456.

Hamilton, I. M. and Ligocki, I. Y. 2012. The extended personality: indirect effects of behavioural syndromes on the behaviour of others in a group-living cichlid. - Anim. Behav. 84: 659-664.

Hamilton, W. D. 1964. The genetical evolution of social behaviour. I. - J. Theor. Biol. 7: 1-16.

Hart, S. P. et al. 2016. How variation between individuals affects species coexistence. - Ecol. Lett. 19: 825-838.

Helms Cahan, S. 2001. Cooperation and conflict in ant foundress associations: insights from geographical variation. - Anim. Behav. 61: 819-825.

Helms, K. R. and Helms Cahan, S. 2012. Large-scale regional variation in cooperation and conflict among queens of the desert ant Messor pergandei. - Anim. Behav. 84: 499-507.

Hochberg, M. E. et al. 2003. Socially mediated speciation. - Evolution 57: 154-158.

Holland, J. N. et al. 2005. Mutualisms as consumer-resource interactions. - In: Ecology of predator-prey interactions. Oxford Univ. Press, pp. 17-33.

Ihara, Y. 2011. Evolution of culture-dependent discriminate sociality: a gene-culture coevolutionary model. - Phil. Trans. R. Soc. B 366: 889-900.

Ito, K. et al. 2017. The evolution of cooperation by negotiation in a noisy world. - J. Evol. Biol. 30: 603-615.

Jensen, J. L. W. V. 1906. Sur les fonctions convexes et les inégalités entre les valeurs moyennes. - Acta Math. 30: 175-193.

Johnstone, R. A. and Bshary, R. 2008. Mutualism, market effects and partner control. - J. Evol. Biol. 21: 879-888.

Jones, E. I. et al. 2012. The fundamental role of competition in the ecology and evolution of mutualisms. - Ann. N. Y. Acad. Sci. 1256: 66-88.

Karst, J. et al. 2008. The mutualism-parasitism continuum in ectomycorrhizas: a quantitative assessment using meta-analysis. Ecology 89: 1032-1042.

Komdeur, J. 2006. Variation in individual investment strategies among social animals. - Ethology 112: 729-747. 
Kraemer, S. A. and Velicer, G. J. 2014. Social complementation and growth advantages promote socially defective bacterial isolates. - Proc. R. Soc. B 281: 20140036.

Lee, A. E. et al. 2016. Information use and resource competition: an integrative framework. - Proc. R. Soc. B 283: 20152550.

Lehtonen, J. and Kokko, H. 2012. Positive feedback and alternative stable states in inbreeding, cooperation, sex roles and other evolutionary processes. - Phil. Trans. R. Soc. B 367: 211-221.

Liu, M. et al. 2020. Social rank modulates how environmental quality influences cooperation and conflict within animal societies. - Proc. R. Soc. B 287: 20201720.

Lynch, M. and Walsh, B. 1998. Genetics and analysis of quantitative traits. - Sinauer, Sunderland, MA.

Marjanovic, J. et al. 2018. Modelling the co-evolution of indirect genetic effects and inherited variability. - Heredity 121: 631-647.

Marshall, H. H. et al. 2016. Variable ecological conditions promote male helping by changing banded mongoose group composition. - Behav. Ecol. 27: 978-987.

McAuliffe, K. et al. 2015. When cooperation begets cooperation: the role of key individuals in galvanizing support. - Phil. Trans. R. Soc. B 370: 20150012.

McNamara, J. M. and Leimar, O. 2010. Variation and the response to variation as a basis for successful cooperation. - Phil. Trans. R. Soc. B 365: 2627-33.

McNamara, J. M. et al. 2004. Variation in behaviour promotes cooperation in the Prisoner's Dilemma game. - Nature 428: $745-748$.

Menzel, F. and Blüthgen, N. 2010. Parabiotic associations between tropical ants: equal partnership or parasitic exploitation? - J. Anim. Ecol. 79: 71-81.

Michod, R. E. 1997. Cooperation and conflict in the evolution of individuality. I. Multilevel selection of the organism. - Am. Nat. 149: 607-645.

Michod, R. E. and Nedelcu, A. M. 2003. On the reorganization of fitness during evolutionary transitions in individuality. Integr. Comp. Biol. 43: 64-73.

Michod, R. E. and Roze, D. 2001. Cooperation and conflict in the evolution of multicellularity. - Heredity 86: 1-7.

Milles, A. et al. 2020. Intraspecific trait variation in personalityrelated movement behavior promotes coexistence. - Oikos 129: 1441-1454.

Moher, D. et al. 2009. Preferred reporting items for systematic reviews and meta-analyses: the PRISMA statement. - PLoS Med. 6: e1000097.

Montesinos-Navarro, A. et al. 2017. Network structure embracing mutualism-antagonism continuums increases community robustness. - Nat. Ecol. Evol. 1: 1661-1669.

Moran, N. P. et al. 2017. Weaving animal temperament into food webs: implications for biodiversity. - Oikos 126: 917-930.

Morris, W. F. et al. 2007. Direct and interactive effects of enemies and mutualists on plant performance: a meta-analysis. - Ecology 88: 1021-1029.

Mougi, A. and Kondoh, M. 2012. Diversity of interaction types and ecological community stability. - Science 337: 349-351.

Müller, C. et al. 2020. The power of infochemicals in mediating individualized niches. - Trends Ecol. Evol. 35: 981-989.

Nicotra, M. L. et al. 2009. A hypervariable invertebrate allodeterminant. - Curr. Biol. 19: 583-589.

Niepoth, N. et al. 2018. Symbiont interactions with non-native hosts limit the formation of new symbioses. - BMC Evol. Biol. 18: 27.
Nonacs, P. and Kapheim, K. M. 2007. Social heterosis and the maintenance of genetic diversity. - J. Evol. Biol. 20: 2253-2265.

Nussey, D. H. et al. 2007. The evolutionary ecology of individual phenotypic plasticity in wild populations. - J. Evol. Biol. 20: 831-844.

Paaby, A. B. and Rockman, M. V. 2014. Cryptic genetic variation: evolution's hidden substrate. - Nat. Rev. Genet. 15: 247-258.

Perez-Barberia, F. J. et al. 2015. State-space modelling of the drivers of movement behaviour in sympatric species. - PLoS One 10: e0142707.

Pesendorfer, M. B. et al. 2016. Competing for seed dispersal: evidence for the role of avian seed hoarders in mediating apparent predation among oaks. - Funct. Ecol. 31: 622-631.

Pinter-Wollman, N. et al. 2016. The effect of keystone individuals on collective outcomes can be mediated through interactions or behavioral persistence. - Am. Nat. 188: 240-252.

Platt, M. L. et al. 2016. Adaptations for social cognition in the primate brain. - Phil. Trans. R. Soc. B 371: 20150096.

Rainey, P. B. and Kerr, B. 2010. Cheats as first propagules: a new hypothesis for the evolution of individuality during the transition from single cells to multicellularity. - BioEssays 32: 872-880.

Ranta, E. et al. 1992. Size matters when three-spined sticklebacks go to school. - Anim. Behav. 43: 160-162.

Ranta, E. et al. 1993. Competition versus cooperation: success of individuals foraging alone and in groups. - Am. Nat. 142: 42-58.

Rathke, E. M. et al. 2017. Age-dependent change of coalitionary strategy in male Barbary macaques. - Prim. Biol. 4: 1-7.

Riebli, T. et al. 2012. Behavioural type, status and social context affect behaviour and resource allocation in cooperatively breeding cichlids. - Anim. Behav. 84: 925-936.

Roberts, G. 1998. Competitive altruism: from reciprocity to the handicap principle. - Proc. R. Soc. B 265: 427-431.

Rodrigues, A. M. and Gardner, A. 2013. Evolution of helping and harming in heterogeneous groups. - Evolution 67: 2284-2298.

Rodriguez-Rodriguez, M. C. et al. 2017. Functional consequences of plant-animal interactions along the mutualism-antagonism gradient. - Ecology 98: 1266-1276.

Sachs, J. L. 2013. Origins, evolution and breakdown of bacterial symbiosis. - In: Levin, S. A. (ed.), Encyclopedia of biodiversity, 2nd edn. Academic Press, pp. 637-644.

Sachs, J. L. and Simms, E. L. 2006. Pathways to mutualism breakdown. - Trends Ecol. Evol. 21: 585-592.

Sachs, J. L. and Wilcox, T. P. 2006. A shift to parasitism in the jellyfish symbiont Symbiodinium microadriaticum. - Proc. R. Soc. B 273: 425-429.

Sachs, J. L. et al. 2004. The evolution of cooperation. - Q. Rev. Biol. 79: 135-160.

Savage, J. L. et al. 2013. Intra-group relatedness affects parental and helper investment rules in offspring care. - Behav. Ecol. Sociobiol. 67: 1855-1865.

Schoepf, I. and Schradin, C. 2013. Endocrinology of sociality: comparisons between sociable and solitary individuals within the same population of African striped mice. - Horm. Behav. 64: 89-94.

Schradin, C. et al. 2009. Testosterone levels in dominant sociable males are lower than in solitary roamers: physiological differences between three male reproductive tactics in a sociably flexible mammal. - Am. Nat. 173: 376-388.

Schuppe, E. R. et al. 2016. The social context of a territorial dispute differentially influences the way individuals in breeding pairs coordinate their aggressive tactics. - Behav. Ecol. Sociobiol. 70: 673-682. 
Sih, A. et al. 2009. Social network theory: new insights and issues for behavioral ecologists. - Behav. Ecol. Sociobiol. 63: 975-988.

Sinervo, B. et al. 2007. Models of density-dependent genic selection and a new rock-paper-scissors social system. - Am. Nat. 170: 663-80.

Singh, M. and Boomsma, J. J. 2015. Policing and punishment across the domains of social evolution. - Oikos 124: 971-982.

Smith, B. R. and Blumstein, D. T. 2008. Fitness consequences of personality: a meta-analysis. - Behav. Ecol. 19: 448-455.

Smith, M. F. et al. 2019. Capuchin monkeys (Sapajus [Cebus] apella) play Nash equilibria in dynamic games, but their decisions are likely not influenced by oxytocin. - Am. J. Primatol. 81: e22973.

Song, Z. and Feldman, M. W. 2013. Plant-animal mutualism in biological markets: evolutionary and ecological dynamics driven by non-heritable phenotypic variance. - Theor. Popul. Biol. 88: 20-30.

Speed, M. P. and Franks, D. W. 2014. Antagonistic evolution in an aposematic predator-prey signaling system. - Evolution 68: 2996-3007.

Stopka, P. and Johnson, D. D. P. 2012. Host-parasite dynamics lead to mixed cooperative games. - Folia Zool. 61: 233-238.

Surbeck, M. et al. 2017. Male reproductive skew is higher in bonobos than chimpanzees. - Curr. Biol. 27: R640-R641.

Székely, T. et al. 2006. Sexual conflict, ecology and breeding systems in shorebirds. - BioScience 56: 801.

Székely, T. et al. 2007. Sexual conflict over parental care: a case study of shorebirds. - J. Ornithol. 148: 211-217.

Takahashi, L. S. et al. 2017. Turn-taking ceremonies in a colonial seabird: does behavioral variation signal individual condition? - Auk 134: 530-541.

Thomas, M. J. et al. 2016. Ontogenetic shifts in a freshwater cleaning symbiosis: consequences for hosts and their symbionts. Ecology 97: 1507-17.

Thompson, J. N. 1988. Variation in interspecific interactions. Annu. Rev. Ecol. Syst. 19: 65-87.

Tilman, D. et al. 1998. Diversity-stability relationships: statistical inevitability or ecological consequence? - Am. Nat. 151:277-282.

Trillmich, F. 2010. Parental care: adjustments to conflict and cooperation. - In: Kappeler, P. (ed.), Animal behaviour: evolution and mechanisms. Springer, pp. 267-298.

Trillmich, F. et al. 2018. Understanding the evolution of personality requires the study of mechanisms behind the development and life history of personality traits. - Biol. Lett. 14: 20170740.
Uitdehaag, J. C. 2011. Bet hedging based cooperation can limit kin selection and form a basis for mutualism. - J. Theor. Biol. 280: 76-87.

Uriarte, M. and Menge, D. 2018. Variation between individuals fosters regional species coexistence. - Ecol. Lett. 21: 1496-1504.

van Dijk, R. E. et al. 2010. Sexual conflict predicts morphology and behavior in two species of penduline tits. - BMC Evol. Biol. 10: 107.

van Dijk, R. E. et al. 2012. Individual variation and the resolution of conflict over parental care in penduline tits. - Proc. R. Soc. B 279: 1927-36.

Violle, C. et al. 2012. The return of the variance: intraspecific variability in community ecology. - Trends Ecol. Evol. 27: 244-252.

Vorburger, C. et al. 2013. Comparing constitutive and induced costs of symbiont-conferred resistance to parasitoids in aphids. - Ecol. Evol. 3: 706-713.

Walker, F. M. et al. 2007. Does soil type drive social organization in southern hairy-nosed wombats? - Mol. Ecol. 16: 199-208.

Wenseleers, T. and Ratnieks, F. L. W. 2006. Enforced altruism in insect societies. - Nature 444: 50-50.

West, S. A. et al. 2007. Social semantics: altruism, cooperation, mutualism, strong reciprocity and group selection. - J. Evol. Biol. 20: 415-432.

Westgate, M. J. 2019. revtools: an R package to support article screening for evidence synthesis. - Res. Synth. Methods 10: 606-614.

Westneat, D. F. et al. 2015. The biology hidden inside residual within-individual phenotypic variation. - Biol. Rev. 90: 729-743.

Wey, T. W. and Blumstein, D. T. 2010. Social cohesion in yellowbellied marmots is established through age and kin structuring. - Anim. Behav. 79: 1343-1352.

Wey, T. W. et al. 2019. Transitivity and structural balance in marmot social networks. - Behav. Ecol. Sociobiol. 73: 1-13.

Whitehead, H. 2017. Gene-culture coevolution in whales and dolphins. - Proc. Natl Acad. Sci. USA 114: 7814-7821.

Whiteman, E. A. and Côté, I. M. 2004. Dominance hierarchies in group-living cleaning gobies: causes and foraging consequences. - Anim. Behav. 67: 239-247.

Wolf, M. and Weissing, F. J. 2010. An explanatory framework for adaptive personality differences. - Phil. Trans. R. Soc. B 365: 3959-3968.

Yuan, Z. L. et al. 2011. From pattern to process: species and functional diversity in fungal endophytes of Abies beshanzuensis. Fungal Biol. 115: 197-213. 